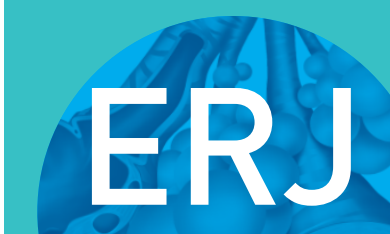

open research

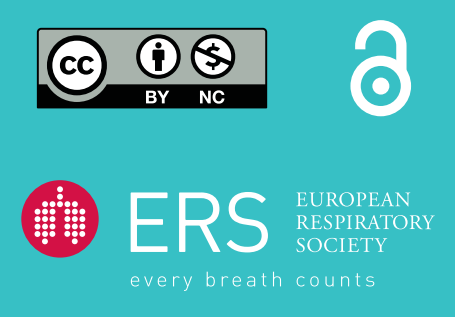

\section{Revisiting the association between temperature and COVID-19 transmissibility across 117 countries}

\author{
To the Editor:
}

The association between ambient temperature and coronavirus disease 2019 (COVID-19) transmissibility has important implications for containing the disease's spread, yet it is still highly debated. Using a dataset including 65 Chinese cities, YAO et al. [1] found no significant association between COVID-19 transmissibility and temperature or ultraviolet (UV) radiation. Using a dataset including 154 Chinese cities, RAN et al. [2] reran the analyses and found a nonlinear negative association between temperature and COVID-19 transmissibility.

While these findings are important in understanding the epidemic pattern of COVID-19, they are not without limitations. First, as the major outbreaks in China ended before April 2020, these studies did not capture the association between temperature and COVID-19 transmissibility in a warmer context. The mean temperatures were below $15^{\circ} \mathrm{C}$ and $10^{\circ} \mathrm{C}$ for $91 \%$ and $80 \%$ of the cities studied by RAN et al. [2], and for $89 \%$ and $81 \%$ of the cities studied by $\mathrm{YAO}_{\mathrm{AO}}$ et al. [1], respectively. YAO et al. [1] suspected a stronger negative association might be observed in a warmer context, but could not test this hypothesis based on their dataset. Second, both analyses were based on data from Chinese cities between December 2019 and February 2020. This results in a relatively narrow geographic corridor and temperature range. Third, many Chinese cities had only a few cases. For low case numbers, the variance across cities could be large and may lead to less precise estimates of the relationship. Fourth, these studies did not control for important confounders such as travel connections, testing intensity, and the distance to Wuhan, the original epicentre of the pandemic.

To complement these studies and add further evidence, we used global data to examine the relationship between temperature and the spread of COVID-19, controlling for several important confounding factors. The global dataset allowed us to capture a broader range of temperatures, more cases within a given country (we included countries with more than 100 cases), and a warmer average temperature across countries [3]. We regressed the prevalence of COVID-19 (logarithmically transformed) at the country level during April against the average temperature within the country at that time. Choosing April as our period of observation amounts to a reasonable compromise between having enough datapoints and capturing an early enough stage of the pandemic for results not to be exceedingly influenced by policy decisions and for cumulative infections having arisen under the same seasonal and climate conditions within countries. As control variables we included: 1) data on air travel [4] and the distance from Wuhan [5] to capture important international transmission patterns of COVID-19; 2) vehicle concentration [6] and urbanisation [4] to capture transmission patterns of COVID-19 within a country [7]; 3) testing intensity [8] to control for policy responses against the spread of COVID-19 and also for the detection bias in cross-country comparisons [9]; 4) cell phone usage [4] to control for the dissemination of information (e.g. on behaviour change for COVID-19 prevention [7]); and 5) income [4] to control for economic activity and the availability of resources to contain the spread of COVID-19.

$@$ ERSpublications

There is a robust and significant negative association between \#COVID19 transmissibility and ambient temperature at the country level. An increase of $1^{\circ} \mathrm{C}$ in temperature is associated with a decrease in the prevalence of COVID-19 by $\sim 5.4 \%$. https://bit.ly/32OTBiS

Cite this article as: Chen S, Prettner K, Cao B, et al. Revisiting the association between temperature and COVID-19 transmissibility across 117 countries. ERJ Open Res 2020; 6: 00550-2020 [https:// doi.org/10.1183/23120541.00550-2020].

Copyright $\odot$ ERS 2020. This article is open access and distributed under the terms of the Creative Commons Attribution NonCommercial Licence 4.0. 
We first used a log-linear regression model akin to that of YAO et al. [1] and RAN et al. [2]. Then we performed regressions with quadratic and cubic specifications and tested for log-linearity by 1) assessing the p-values of quadratic and cubic terms, which were insignificant; and 2) comparing the Akaike information criterion (AIC) and Bayesian information criterion (BIC) across all specifications. AIC and BIC criteria are used for model selection among a finite set of models, where the model with the lowest AIC or BIC is preferred. Our results indicate the best fit for a log-linear specification. Finally, 3) we tested for log-linearity by applying a likelihood ratio test on the log-linear specification versus the specification with a quadratic term, which did not reject the null hypothesis of a log-linear relationship. All the results therefore suggested that there were no significant departures from log-linearity (figure 1). In line with RAN et al. [2], the AIC did not shrink when adding higher-order terms.

Unlike YAO et al. [1], who found no association, our results suggest a robust and significant inverse loglinear relationship between temperature and COVID-19 transmissibility. Our findings are broadly consistent with the findings from RAN et al. [2], suggesting a more evidently negative association between temperature and COVID-19 transmissibility in a warmer context. In the preferred multivariable log-linear specification, an increase of $1^{\circ} \mathrm{C}$ in temperature is associated with a decrease in the prevalence of COVID-19 by $\sim 5.4 \%$. Our results therefore suggest that COVID-19's transmissibility is likely to be lower when the weather is warmer. This is consistent with many other viral acute respiratory tract infections, such as influenza A and $\mathrm{B}$, rhinovirus, respiratory syncytial virus, adenovirus, metapneumovirus, and other coronaviruses, which are climate dependent and share similar seasonal patterns [10]. Thus, countries that try to contain the spread of COVID-19 by different measures in spring and summer may find this easier than in autumn and winter, when (keeping everything else equal) lower temperature increases the transmissibility of the virus.

However, this study is not without limitations. First, our analysis is based on average temperature within the country at that time. While our results are complementary to city-level findings within one country, it would be ideal to have city-level data in a cross-country study as well because some big countries may have a wide range of temperature within the country. Second, we used air travel as a control variable. However, in some countries, flights in April were almost all cancelled. Yet, the relationship is significant and the conclusions remained unchanged irrespective of whether we include air travel as a control variable or not. Third, although we have improved previous studies by adding important control variables, there may still be other unobserved confounders. Nevertheless, the main value added by this study is to complement earlier results that were based on within-country variation with cross-country variation. We
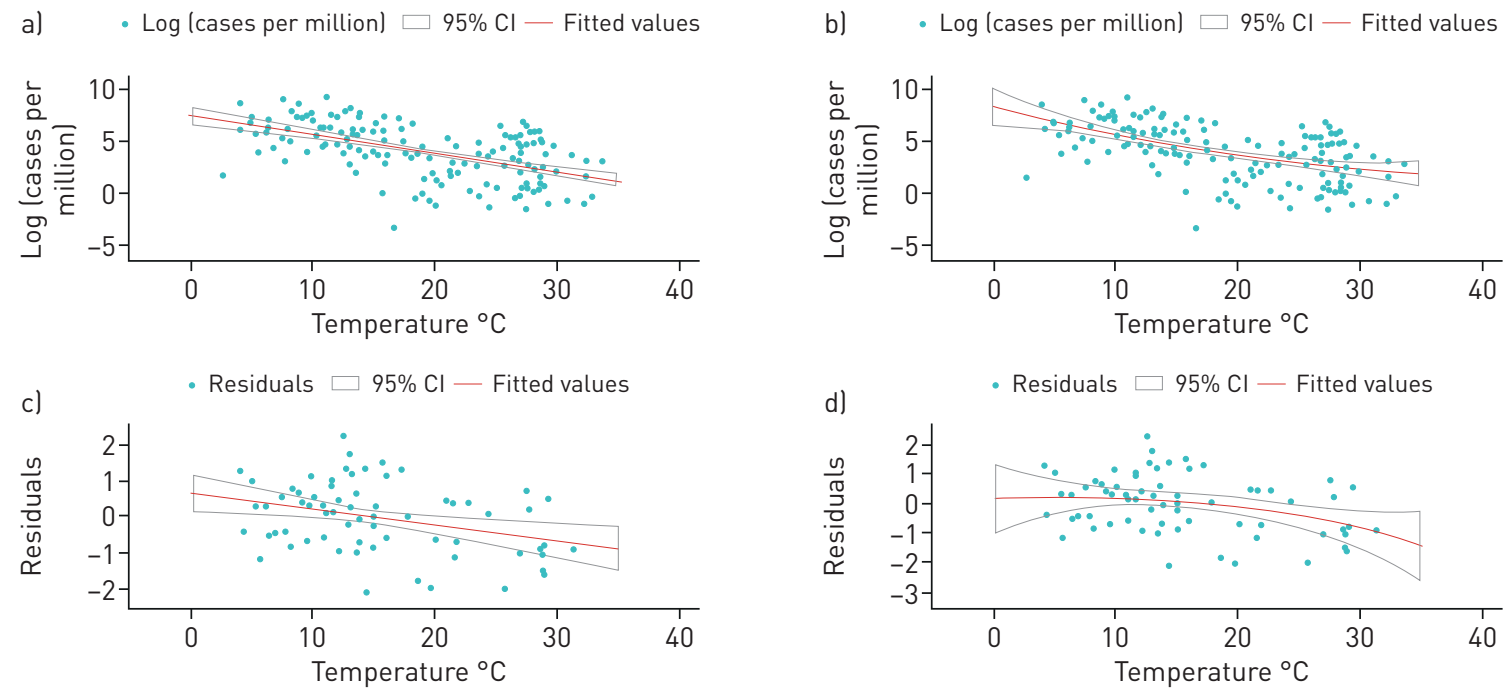

FIGURE 1 Scatterplots of the logarithm of cases per million inhabitants against temperature. Association between temperature and COVID-19 transmissibility (natural logarithm of cases per million inhabitants) across 117 countries. a) Bivariate log-linear regression of COVID-19 prevalence on temperature ( $p$-value $=<0.0001$; Akaike information criterion $(A I C)=439.4$; Bayesian information criterion (BIC) $=444.9$; R-squared=0.32). b) Regression including a quadratic specification for temperature ( $p$-value of linear term=0.008; $p$-value of quadratic term=0.191; $A I C=439.6 ; \quad B I C=447.9 ; R$-squared=0.33). c) Multiple log-linear regression of COVID-19 prevalence on temperature ( $p$-value=0.005; $A I C=189.9 ; B I C=209.5 ; R$-squared=0.72). d) Multiple regression including a quadratic specification for temperature ( $p$-value of linear term=0.494; $\mathrm{p}$-value of quadratic term $=0.201 ; \mathrm{AIC}=189.9 ; \mathrm{BIC}=211.7 ; \mathrm{R}$-squared=0.73). Only countries with more than 100 cases reported as of April 10,2020 were included. In all the multiple regressions we control for "air travel" (i.e. the number of air passengers per capita in a country), "distance from Wuhan" (i.e. the distance of the capital city of a country from Wuhan, the original epicentre of the epidemic, in thousands of kilometres), "vehicle concentration" (i.e. the number of registered vehicles per capita), "urbanisation" (i.e. the percentage of the population living in cities), "testing intensity" (i.e. the number of tests per confirmed case), "cell phone usage" (i.e. the number of cell phones per capita), and "income" (i.e. the purchasing power adjusted per capita gross domestic product in a countryl. 
show that the central result of a negative association between temperature and COVID-19 prevalence remains valid in such a setting.

This study shows that there is a robust and significant negative association between COVID-19 transmissibility and ambient temperature at the country level. Our result is highly significant at the $1 \%$ level ( $p$-value $=0.005$ in the multivariable analysis), which compares with a significance level of $5 \%$ ( $\mathrm{p}$-value $=0.049$ when temperature is over $7^{\circ} \mathrm{C}$ ) in RAN et al. [2]. While this is in line with the results of RAN et al. [2] and we improved upon this study by adding important covariates (McFadden's pseudo-R-squared in their study is $11 \%$ as compared to $72 \%$ in our study), both studies cannot isolate the effect of UV radiation that is correlated with heat and seems to kill the virus quite quickly in experiments, the fact that people spend more time outside if the temperature is warmer, or the enhanced vitamin $\mathrm{D}$ production of the human body in sunnier conditions [3]. We suggest that the causal impact of temperature on COVID-19 transmissibility and the underlying mechanisms such as virus viability, the host immunity, and people's behaviour should be further explored in more in-depth studies.

Simiao Chen $\odot^{1,2,11}$, Klaus Prettner ${ }^{3,11}$, Bin $\mathrm{Cao}^{4}$, Pascal Geldsetzer ${ }^{1,5}$, Michael Kuhn ${ }^{6}$, David E. Bloom ${ }^{7}$, Till Bärnighausen ${ }^{1,2,7,8,12}$ and Chen Wang ${ }^{2,4,9,10,12}$

${ }^{1}$ Heidelberg Institute of Global Health, Heidelberg University Medical School, Heidelberg University, Heidelberg, Germany. ${ }^{2}$ Chinese Academy of Medical Sciences and Peking Union Medical College, Beijing, China. ${ }^{3}$ Vienna University of Economics and Business (WU), Dept of Economics, Vienna, Austria. ${ }^{4}$ Dept of Pulmonary and Critical Care Medicine, Center of Respiratory Medicine, China-Japan Friendship Hospital, Beijing, China. ${ }^{5}$ Division of Primary Care and Population Health, Dept of Medicine, Stanford University, Stanford, CA, USA. ${ }^{6}$ Wittgenstein Centre (IIASA, OeAW, University Vienna), Vienna Institute of Demography, Vienna, Austria. ${ }^{7}$ Dept of Global Health and Population, Harvard T.H. Chan School of Public Health, Boston, MA, USA. ${ }^{8}$ Africa Health Research Institute (AHRI), Somkhele, KwaZulu-Natal, South Africa. ${ }^{9}$ National Clinical Research Center for Respiratory Diseases, Beijing, China. ${ }^{10}$ Chinese Academy of Engineering, Beijing, China. ${ }^{11} \mathrm{Co}$-first authors. ${ }^{12} \mathrm{Co}$-senior authors.

Correspondence: Chen Wang, Chinese Academy of Medical Sciences and Peking Union Medical College, 9 Dongdan Santiao Road, Dongcheng District, Beijing 100730, China. E-mail: wangchen@pumc.edu.cn

Received: 6 Aug 2020 | Accepted after revision: 14 Sept 2020

Support statement: This study was supported by Bill and Melinda Gates Foundation grant Project INV-006261. Funding information for this article has been deposited with the Crossref Funder Registry.

Conflict of interest: None declared.

References

1 Yao Y, Pan J, Liu Z, et al. No Association of COVID-19 transmission with temperature or UV radiation in Chinese cities. Eur Respir J 2020; 55: 2000517.

2 Ran J, Zhao S, Han L, et al. A re-analysis in exploring the association between temperature and COVID-19 transmissibility: an ecological study with 154 Chinese cities. Eur Respir J 2020; 56: 2001253.

3 Chen S, Prettner K, Kuhn M, et al. COVID-19 and climate: global evidence from 117 countries. medRxiv 2020; preprint [https://doi.org/10.1101/2020.06.04.20121863].

4 World Bank. World Development Indicators. 2020. https://databank.worldbank.org/source/world-developmentindicators Date last updated: 16 September 2020. Date last accessed: 11 April 2020.

5 Google Map Developers. Distance calculator tool. www.mapdevelopers.com/distance_finder.php

6 World Health Organization. Global status report on road safety 2018: summary. Geneva, World Health Organization, 2018.

7 Chen S, Yang J, Yang W, et al. COVID-19 control in China during mass population movements at New Year. Lancet 2020; 395: 764-766.

8 Our World in Data. Coronavirus (COVID-19) Testing. https://ourworldindata.org/covid-testing Date last updated: 6 October 2020. Date last accessed: 11 April 2020.

9 Chen S, Zhang Z, Yang J, et al. Fangcang shelter hospitals: a novel concept for responding to public health emergencies. Lancet 2020; 395: 1305-1314.

10 Stewart PDS. Seasonality and selective trends in viral acute respiratory tract infections. Med Hypotheses 2016; 86: $104-119$. 